\title{
3D Stent Recovery from One X-Ray Projection
}

\author{
Stefanie Demirci ${ }^{1, \star}$, Ali Bigdelou ${ }^{1, \star}$, Lejing Wang ${ }^{1}$, Christian Wachinger $^{1}$, \\ Maximilian Baust ${ }^{1}$, Radhika Tibrewal ${ }^{1}$, Reza Ghotbi $^{2}$, \\ Hans-Henning Eckstein ${ }^{3}$, and Nassir Navab ${ }^{1}$ \\ 1 Computer Aided Medical Procedures, Technische Universität München, Germany \\ \{demirci, bigdelou, wangle, wachinge, baust, rtibrewal, navab\}@cs. tum.edu \\ 2 Vascular Surgery, Klinikum München-Pasing, Germany \\ reza.ghotbi@krankenhaus-pasing. de \\ 3 Department of Vascular Surgery, Klinikum rechts der Isar, Germany \\ h.h.eckstein@lrz.tum.de $\mathrm{d}^{\star \star}$
}

\begin{abstract}
In the current clinical workflow of endovascular abdominal aortic repairs (EVAR) a stent graft is inserted into the aneurysmatic aorta under 2D angiographic imaging. Due to the missing depth information in the X-ray visualization, it is highly difficult in particular for junior physicians to place the stent graft in the preoperatively defined position within the aorta. Therefore, advanced $3 \mathrm{D}$ visualization of stent grafts is highly required. In this paper, we present a novel algorithm to automatically match a 3D model of the stent graft to an intraoperative $2 \mathrm{D}$ image showing the device. By automatic preprocessing and a globalto-local registration approach, we are able to abandon user interaction and still meet the desired robustness. The complexity of our registration scheme is reduced by a semi-simultaneous optimization strategy incorporating constraints that correspond to the geometric model of the stent graft. Via experiments on synthetic, phantom, and real interventional data, we are able to show that the presented method matches the stent graft model to the $2 \mathrm{D}$ image data with good accuracy.
\end{abstract}

\section{Introduction}

Due to its minimally invasive nature, endovascular aortic repair (EVAR) has replaced the conventional open surgery as preventive treatment procedure for abdominal aortic aneurysms (AAA). In contrast to a complete opening of the patient's abdomen, a stent graft is inserted through one femoral artery into the aneurysmatic aorta excluding the aneurysm sack from the circulation and reducing the pressure on the aortic wall. Once completely unfolded, misplacements of the stent graft may lead to life-threatening emergency surgeries if vitally important organs are cut-off from blood supply.

\footnotetext{
* Joint first authors.

** We are thankful to Simon Weidert (Klinikum Innenstadt, Ludwig-MaximilianUniversität München, Germany) and Asbjorn Odegard (St. Olavs Hospital Trondheim, Norway) for providing image data.
}

G. Fichtinger, A. Martel, and T. Peters (Eds.): MICCAI 2011, Part I, LNCS 6891, pp. 178 $185,2011$.

(C) Springer-Verlag Berlin Heidelberg 2011 


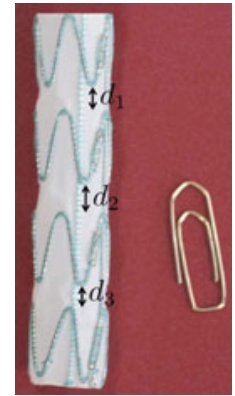

Fig. 1. Tubular aortic stent graft
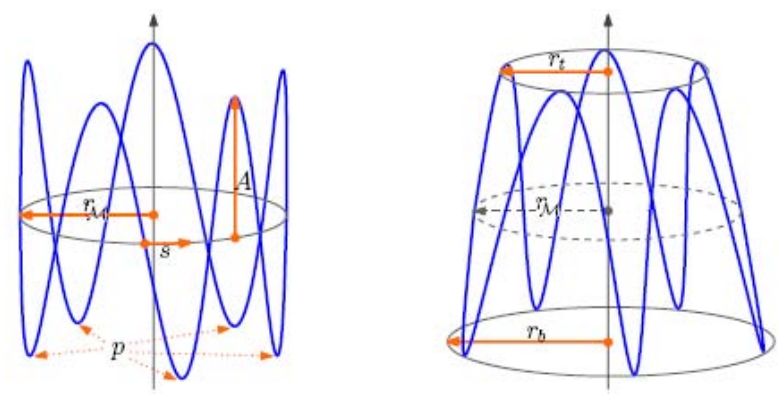

During the intervention, the catheter and stent position is only visualized in 2D X-ray views. This makes it highly difficult for physicians to place the stent graft in the predefined position. A virtual visualization of the unfolding stent graft within the CTA volume would provide the physician a $3 \mathrm{D}$ view of the current situation. Such solutions would decrease both the need for extensive use of contrast agent and the radiation dose. Whereas 2D-3D registration systems for endovascular interventions are already available 68 , a visualization of the stent graft in a $3 \mathrm{D}$ patient scan still requires a method for stent graft detection in $2 \mathrm{D}$ and correct backprojection into $3 \mathrm{D}$.

Existing publications on stent graft segmentation in $3 \mathrm{D}$ volumes $7 / 4$ all employ intensity based thresholding. There is, to the best of our knowledge, no shape-based approach for extraction of aortic stent grafts in 3D or 2D.

In this paper, we present a novel algorithm to match a $3 \mathrm{D}$ model of the stent graft to an intraoperative 2D image showing the device.

\section{Method}

\subsection{Stent Model}

We define the stent segment model to be the curve $\mathcal{M}_{i}(\mathbf{x})=\left(a_{i}(\mathbf{x}), b_{i}(\mathbf{x}), c_{i}(\mathbf{x})\right)$ consisting of the set of parametric equations

$$
\begin{aligned}
a_{i}(\mathbf{x}) & =r_{\mathcal{M}, i}(\mathbf{x}) \cos (\mathbf{x}) \\
b_{i}(\mathbf{x}) & =r_{\mathcal{M}, i}(\mathbf{x}) \sin (\mathbf{x}) \\
c_{i}(\mathbf{x}) & =A_{i} \sin \left(p_{i} \mathbf{x}+s_{i}\right)
\end{aligned}
$$

with amplitude $A_{i}$ specifying the height of the segment, period $p_{i}$ equal to the number of peaks, and phase shift $s_{i}$ merely shifting the starting point. The radius of the stent segment model is calculated by

$$
r_{\mathcal{M}, i}(\mathbf{x})=\left(1-\frac{\sin \left(p_{i} \mathbf{x}+1\right)}{2}\right) r_{i}^{t}+\frac{\sin \left(p_{i} \mathbf{x}\right)+1}{2} r_{i}^{b}
$$




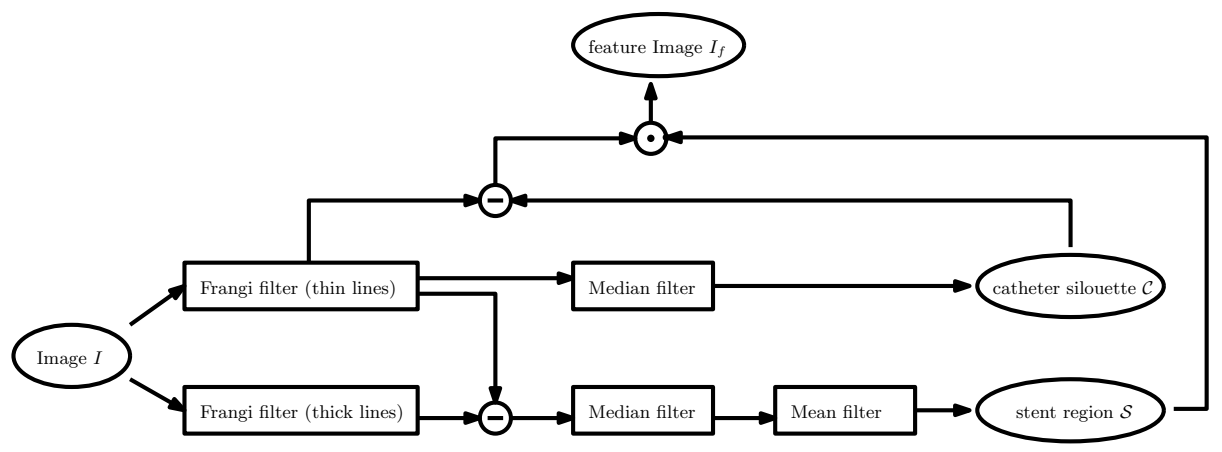

Fig. 3. Preprocessing scheme for an automatic computation of the feature image $I_{f}$

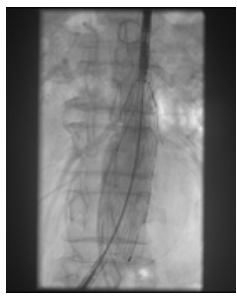

(a) $I$

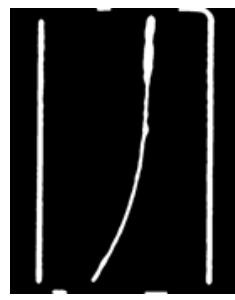

(b) $\mathcal{C}$

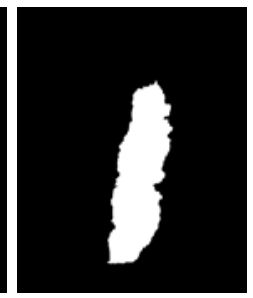

(c) $\mathcal{S}$

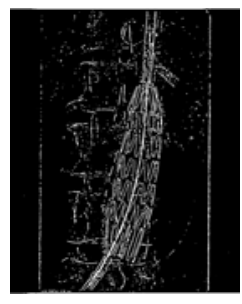

(d) $I_{\text {wires }}$

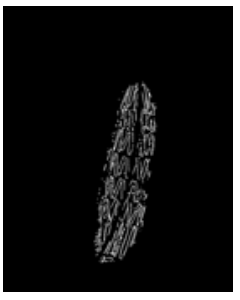

(e) $I_{f}$

Fig. 4. Visualization of automatic feature extraction procedure

with $r_{i}^{t}$ and $r_{i}^{b}$ representing the upper and lower radius as visualized in Fig. 2, The entire stent graft is defined as the set of $l$ segments $\mathcal{M}=\left\{\mathcal{M}_{1}, \ldots, \mathcal{M}_{l}\right\}$.

\subsection{Automatic Feature Extraction}

For an improved performance, we first apply a preprocessing procedure on image $I$ (see Fig. 4(a) in order to extract a region of interest and highlight the wire structure. Additional to the wires of the stent graft, the guide wire which is used as navigation support to the physician is also visible in the interventional X-ray images. In order for our matching algorithm to not get stuck in these outliers, we need to eliminate these pixels.

Fig. 3 displays our preprocessing scheme. For obtaining the catheter silhouette image $\mathcal{C}$ (Fig. 4(b)], we employ the Frangi filter [2] for scales $5-6$ followed by a median filtering for noise removal in order to capture the catheter pixels.

The stent region $\mathcal{S}$ (Fig. 4(c) is extracted similarly. First, we subtract thick curvilinear structures from thin curvilinear structures (Frangi filter for scale 2) for only highlighting the stent wires in $I_{\text {wires }}$ (Fig.4(d). Subsequent employment of a median filter for noise removal and mean filter for dominant region extraction leads to the desired image region that contains the stent graft. Instead of the Frangi filter, any filter can be used that highlights curvilinear structures of selected sizes. 
In the end, we subtract the catheter pixels $\mathcal{C}$ from image $I_{\text {wires }}$ and mask the result with $\mathcal{S}$. The resulting feature image $I_{f}$ is displayed in Fig. 4(e) In order to provide a distance measure for the following registration procedure, we calculate distance map $D_{I_{f}}$ on feature image $I_{f}$.

\subsection{Stent-Model-to-Image Registration}

A projection is necessary to map the stent model $\mathcal{M}$ to the $2 \mathrm{D}$ image coordinate system of $I$. Similar to 2D-3D image registration, the projection transformation $\mathbf{P}=K[R \mid t]$ consists of the 6 -DOF extrinsic parameters $[R \mid t]$ for rotation and translation of the 3D volume and the 4-DOF intrinsic camera parameter $K$ of the pinhole camera model [3]. For the following considerations, we assume the camera matrix $K$ to be given by the interventional angio system.

Using all transformation and model parameters together as

$$
\mathbf{p}=\left\{\mathbf{p}_{1}, \ldots, \mathbf{p}_{l}\right\}, \quad \mathbf{p}_{\mathbf{i}}=\left\{R_{i}, t_{i}, r_{i}^{t}, r_{i}^{b}, A_{i}\right\}
$$

the registration problem for the entire stent model is given by

$$
\hat{\mathbf{p}}=\arg \min _{\mathbf{p}} \sum_{i=1}^{l} \sum_{x \in \mathcal{M}_{i}} D_{I_{f}}\left(T_{\mathbf{p}_{i}}(x)\right)
$$

where $T_{\mathbf{p}_{i}}(x)$ is a projection of point $x$ of the $3 \mathrm{D}$ segment model $\mathcal{M}_{i}$ using parameters $\mathbf{p}_{i}$. This equals a simultaneous registration of all stent segment models introducing a parameter space of dimension $l \times 9$. Considering that conventional abdominal aortic stent grafts consist of several segments, the cost for optimization increases rapidly. Another drawback is the presence of many local minima in the costfunction plot corresponding to each one of all displayed stent segments.

In order to reduce the complexity in our registration procedure, we employ a semi-simultaneous optimization framework based on the idea of Sidorov et al. 9]. Instead of optimizing all parameters for all segments at once, we optimize the parameters of one segment for a certain number of iterations and then move to the next randomly chosen segment. By applying this strategy, we implicitly make use of the tubular appearance of the stent graft.It requires however a fairly good initial guess of the position of the stent in the image which we try to achieve by separating the pose estimation process into a global and a local step.

Global registration. Here, we solve for the overall orientation of all segments in order to be very close for the local calculations. The global pose of the entire stent graft model $\mathcal{M}$ is defined by the global parameters $\mathbf{p}_{\text {global }}=\left\{K, R_{\text {global }}, t_{\text {global }}\right\}$. Assuming that $K$ is given, a good initial guess for the remaining global parameters is achieved by computing a principal component analysis and subsequent region operations on the stent region $\mathcal{S}$.

Accordingly, let now $\mathbf{p}_{i}=\left\{\mathbf{v}_{i}, \mathbf{t}_{i}, r_{i}^{t}, r_{i}^{b}, A_{i}\right\}$ define the set of remaining parameters for each single segment $i$, where $\mathbf{v}_{i}=\left[\alpha_{i}, \beta_{i}, \gamma_{i}\right]^{T}$ represents the vector containing the three rotation angles that form rotation matrix $\mathbf{R}_{i}$. Setting 
parameter vectors $\mathbf{p}_{i}$ to initial values with no rotation and translation and predefined approximated values for $A$ and $r^{t}=r^{b}$, we obtain an estimation of the position of each stent segment in the interventional image.

Local registration. In this step, values for each $\mathbf{p}_{i}$ are optimized. Similar to Equ. (6), we define our costfunction for each segment $i(i=1, \ldots, l)$ as

$$
E\left(\mathbf{p}_{i}\right)=\sum_{x \in \mathcal{M}_{i}} D_{I_{f}}\left(T_{\mathbf{p}_{\text {global }} \circ \mathbf{p}_{i}}(x)\right)+\frac{\omega}{\lambda}\left\langle\phi\left(\mathbf{p}_{i}\right), \phi\left(\mathbf{p}_{i+1}\right)\right\rangle
$$

with

$$
\phi\left(\mathbf{p}_{i}\right)=\left\|\begin{array}{l}
\left|\mathbf{t}_{i}-\mathbf{t}_{i-1}\right|-\mathbf{t}_{i}^{\Delta} \\
\left|\mathbf{v}_{i}-\mathbf{v}_{i-1}\right|-\mathbf{v}_{i}^{\Delta} \\
\left|r_{i}^{t}-r_{i-1}^{b}\right|-r_{i}^{\Delta}
\end{array}\right\|
$$

constraining the change of translation, rotation and radius in between neighboring segments naturally defined by the graft material fixing the stent wires (Fig. 1) that is not stretchable. Therefore respective parameters depend on the predefined distance $d_{i}$ between the segments:

$$
\mathbf{t}_{i}^{\Delta}=\left[\begin{array}{c}
\frac{d_{i}}{2} \\
\frac{d_{i}}{2} \\
\frac{A_{i}}{2}+d_{i}
\end{array}\right] \quad \mathbf{v}_{i}^{\Delta}=\left[\begin{array}{c}
\frac{d_{i}}{\sqrt{1+d_{i}}} \\
\frac{d_{i}}{\sqrt{1+d_{i}}} \\
\frac{s_{i}}{2}
\end{array}\right] \quad r_{i}^{\Delta}=\left|r_{i}^{t}-r_{i}^{b}\right|
$$

$\omega>>0$ represents a weighting factor for the penalization term. We optimize Equ. 7 within a deterministic annealing scheme with temperature $\lambda$ being the distance from the current projected segment to the nearest image feature in $I_{f}$. As the distance and hence the temperature decrease, the penalization term is taken more and more into account. Using this approach our algorithm is also able to capture highly curved stent grafts.

\section{Results and Discussion}

Although our method can be applied to interventional X-ray images without knowing the corresponding matrix $\mathbf{P}$, a thorough validation is only possible in $3 \mathrm{D}$ requiring a correct calibration of the $\mathrm{C}$-arm system. A possible strategy is to acquire projection images of the stent graft from two or more different views, apply our proposed method to each of the images, and compare the resulting 3D models. Therefore it is crucial that either all images are taken simultaneously or no changes to the stent graft have been made in between the different acquisitions. It is very difficult to find interventional images that fulfill these requirements as bi-planar systems are not considered state-of-the-art imaging for endovascular interventions and physicians only rarely acquire two or more images where the stent graft is in the exact same opening stage. Another approach would be to compare the resulting $3 \mathrm{D}$ stent model to a segmentation of the stent in a $3 \mathrm{D}$ volume. This is not possible for interventional cases, as only 
Table 1. Synthetic experiments: RMS errors for amplitude, top and bottom radii averaged over total number of included segments and target registration errors over all landmarks

\begin{tabular}{|l||c|c|c||c|c|}
\hline & $A(\mathrm{~mm})$ & $r^{t}(\mathrm{~mm})$ & $r^{b}(\mathrm{~mm})$ & \# landmarks & mean/median/max TRE $(\mathrm{mm})$ \\
\hline \hline $\mathbf{R}_{3}$ & 0.00 & 0.00 & 0.00 & 12 & $6.29 / 3.86 / 8.65$ \\
$\mathbf{R}_{4}$ & 0.00 & 0.00 & 0.00 & 16 & $6.54 / 4.01 / 7.96$ \\
$\mathbf{R}_{5}$ & 0.00 & 0.04 & 0.02 & 20 & $7.02 / 5.36 / 10.50$ \\
$\mathbf{R}_{6}$ & 0.01 & 0.04 & 0.04 & 24 & $7.39 / 5.81 / 9.91$ \\
\hline
\end{tabular}

pre- and (maybe) postoperative patient volumes are available that either contain no stent graft at all (preoperative) or a fully opened stent graft (postoperative). Due to these difficulties, we decided to quantitatively validate the accuracy of our proposed algorithm on realistic synthetic and phantom data and use in-vivo data to show the interventional impact of our method.

With this paper we intend to present a proof-of-concept for a highly novel approach to enhance intraoperative visualization for EVAR. We would like to emphasize that the proposed method is of linear complexity $\mathcal{O}(n)$ with $n$ being the number of pixels included in one stent segment. Depending on the image resolution, $n$ lies in between 100 and 500. Although all experiments were performed using unoptimized MATLAB code, with an efficient implementation, it is possible to apply the procedure within an interventional setting.

For creating the synthetic data, we digitally produced binary volumes of size $512 \times 512 \times 512$ showing random stent graft models consisting of 3-6 segments, namely $\mathbf{R}_{3}, \mathbf{R}_{4}, \mathbf{R}_{5}, \mathbf{R}_{6}$. By applying realistic deformations to each of the segments and calculating random projections of the volumes, we obtain $2 \mathrm{D}$ images of the stent graft as well as the corresponding ground truth parameter selection.We estimated the average noise level of 5 interventional fluoroscopies showing an unfolded stent graft and added it to the projection images (mean 0.59 and variance 0.98). The registration accuracy measured in target registration error (TRE) in varying number of distributed landamrks, is listed in Table 1. As expected, the errors values are very small and show the overall impact of our method. The sudden change in error values for stent models with 5 and 6 segments are due to the fact that we only employed different top and bottom radii for $\mathbf{R}_{5}, \mathbf{R}_{6}$.

The phantom design is composed of the upper part of a swine's leg in which we inserted a halfly unfolded stent graft parallel to the bone. For guide wire simulation, we integrated a small wire inside the stent graft. In order to prevent deformation changes, we placed the entire phantom in a paper box and filled all empty space with insulating foam. 10 radio-opaque markers were sticked to the outside and inside of the box in a predefined pattern. After acquiring a 3D CT volume, we took X-ray projection images with a mobile interventional C-arm from 3 different angles. Details of the image sizes and transformations are given in Table 2. In absence of an available fixed angio system, we had to calibrate the C-arm by employing the normalized Direct Linear Transform (DLT) algorithm [3] on the corresponding marker positions. Once $\mathbf{P}$ matrices were estimated, our algorithm was applied to all 3 projection images. We compared the recovered 
Table 2. Details for phantom projection images and the Hausdorff distance of recovered $3 \mathrm{D}$ stent model to real stent graft extracted from $\mathrm{CT}$

\begin{tabular}{|c|c|c|c||c|}
\hline & size (pixel) & 1st angle (deg) & 2nd angle (deg) & Hausdorff $(\mathrm{mm})$ \\
\hline \hline $\mathrm{A}$ & $478 \times 422$ & 18.5 & 20.0 & 20.2 \\
$\mathrm{~B}$ & $478 \times 422$ & 0.5 & 15.0 & 25.4 \\
$\mathrm{C}$ & $478 \times 422$ & 108.5 & -4.5 & 15.1 \\
\hline
\end{tabular}

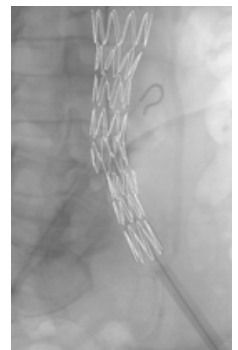

(a)

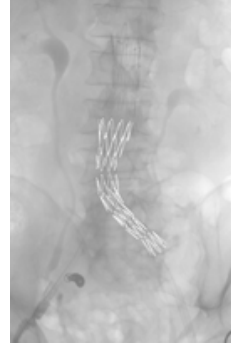

(b)

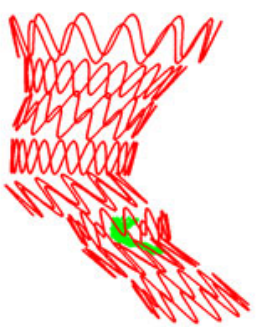

(c)

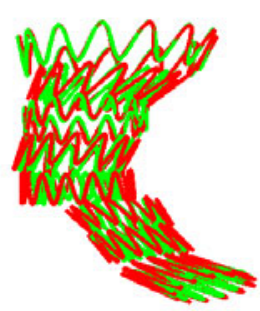

(d)

Fig. 5. Real experiment: (a)-(b) detection results (white) overlayed onto interventional images acquired from two different views, (c) 3D recovery of both stent shapes (green,red) and (d) after applying a common scale to all green segments

3D models to the real stent extracted from CT using a combination of manual outlining and region growing. In order to allow for quantitative evaluation of our results, we backprojected the resulting digital stent models into CT space and created a binary volume of the exact same extent and voxel size as the phantom CT volume. Values for the Hausdorff distance measuring the distance between both shapes for each of the 3 datasets, are given in Table 2 .

For the real experiments, our medical partners kindly provided a set of real interventional fluoroscopy image data (acquired by Siemens AXIOM Artis dTA angiography suite) and corresponding preoperative CTA scans. Provided that the C-arm system is correctly calibrated, the amplitude and top and bottom radii of two corresponding segments acquired from different views must be equal up to a common scaling factor. As the angiography system used by our clinical partners is not biplanar, deformation changes need to be taken care of by the experimental setup. In the lower abdominal part of the human body, the aorta and iliac arteries are not exposed to breathing or other organ specific motion and the pulsatile motion originating from the blood pressure is neglectable [5]. The deformation that is induced by the stent graft itself, however, is very significant [1], but can be eliminated by acquiring both images in the same opening stage of the stent graft. Visual results are given in Fig. 5. For the combined plot of both models (Fig. 5(d), we applied a common scale of 7.91 to each segment's amplitude and top and bottom radii of the stent model shown in red color. The scale value represents the mean ratio of final values for amplitude and top and bottom radii for each of the 8 segments and yields a root mean square error in model point positions of $2.1 \mathrm{~mm}$. 


\section{Conclusion}

In this paper, we presented a novel algorithm to match a $3 \mathrm{D}$ model of the stent graft to an intraoperative 2D image showing the device. It uses automatic preprocessing and a global-to-local approach to abandon any user interaction and still meet the required robustness. Including a semi-simultaneous optimization strategy and constraining the inter-stent-segment relations to correspond to the material property of the stent graft, the complexity of the optimization space could be reduced. The results of performed experiments on synthetic as well as real interventional data show the practical potential of our proposed method.

\section{References}

1. Demirci, S., Manstad-Hulaas, F., Navab, N.: Quantification of aortic deformation after EVAR. In: Miga, M.I., Wong, K.H. (eds.) Medical Imaging: Visualization, Image-Guided Procedures, and Modeling. Proceedings of SPIE. SPIE, vol. 7261, p. $72611 \mathrm{U}(2009)$

2. Frangi, A.F., Niessen, W.J., Vincken, K.L., Viergever, M.A.: Multiscale vessel enhancement filtering. In: Wells, W.M., Colchester, A.C.F., Delp, S.L. (eds.) MICCAI 1998. LNCS, vol. 1496, pp. 130-137. Springer, Heidelberg (1998)

3. Hartley, R., Zisserman, A.: Multiple View Geometry, 2nd edn. University Press, Cambridge (2003)

4. Klein, A., Renema, W., Schultze Kool, L., Slump, C.: Initial steps towards automatic segmentation of the wire frame of stent grafts in ct data. In: 4th Annual Symposium of the IEEE-EMBS Benelux Chapter, pp. 116-119. IEEE-EMBS Benelux Chapter, Enschede (2009)

5. Klein, A., Oostveen, L.J., Greuter, M.J.W., Hoogeveen, Y., Schultze Kool, L.J., Slump, C.H., Renema, W.K.J.: Detectability of motions in aaa with ecg-gated cta: a quantitative study. Med. Phys. 36(10), 4616-4624 (2009)

6. Liao, R., Tan, Y., Sundar, H., Pfister, M., Kamen, A.: An efficient graph-based deformable $2 \mathrm{D} / 3 \mathrm{D}$ registration algorithm with applications for abdominal aortic aneurysm interventions. In: Liao, H., Edwards, P.J., Pan, X., Fan, Y., Yang, G.-Z. (eds.) MIAR 2010. LNCS, vol. 6326, pp. 561-570. Springer, Heidelberg (2010)

7. Mattes, J., Steingruber, I., Netzer, M., Fritscher, K., Kopf, H., Jaschke, W., Schubert, R.: Quantification of the migration and deformation of abdominal aortic aneurysm stent grafts. In: Reinhardt, J.M., Pluim, J.P.W. (eds.) Medical Imaging: Image Processing. Proceedings of SPIE, vol. 6144, p. 61440V. SPIE (2006)

8. Raheem, A., Carrell, T., Modarai, B., Penney, G.: Non-rigid 2D-3D image registration for use in endovascular repair of abdominal aortic aneurysms. In: Bhalerao, A.H., Rajpoot, N.M. (eds.) Proceedings of Medical Image Understanding and Analysis, pp. 153-158. University of Warwick (2010)

9. Sidorov, K., Richmond, S., Marshall, D.: An efficient stochastic approach to groupwise non-rigid image registration. In: IEEE Conference on Computer Vision and Pattern Recognition, pp. 2208-2213. IEEE Press, Los Alamitos (2009) 